\title{
The Femoral Hernia: Some Necessary Additions
}

\author{
Ljubomir S. Kovachev \\ Department of General Surgery, Medical University, Pleven, Bulgaria \\ Email: Iskovachev@hotmail.com
}

Received 28 April 2014; revised 27 May 2014; accepted 26 June 2014

Copyright (C) 2014 by author and Scientific Research Publishing Inc.

This work is licensed under the Creative Commons Attribution International License (CC BY). http://creativecommons.org/licenses/by/4.0/

c) (i) Open Access

\begin{abstract}
Purpose: The anatomic region through which most inguinal hernias emerge is overcrowded by various anatomical structures with intricate relationships. This is reflected by the wide range of anatomic interpretations. Material and Methods: A prospective anatomic study of over 100 fresh cadavers and 47 patients operated on for femoral hernias. Results: It was found that the transversalis fascia did not continue distally into the lymphatic lacuna. Medially this fascia did not reach the lacunar ligament, but was rather positioned above it forming laterally the vascular sheath. Here the fascia participates in the formation of a fossa, which varies in width and depth-the preperitoneal femoral fossa. The results did not confirm the presence of a femoral canal. The distances were measured between the pubic tubercle and the medial margin of the femoral vein, and between the inguinal and the Cooper's ligaments. The results clearly indicate that in women with femoral hernias these distances are much larger. Along the course of femoral hernia exploration we established the presence of three zones that are rigid and narrow. These are the potential sites for femoral hernia incarceration. Conclusion: In the lower infra-inguinal space, where femoral hernias could appear, the integrity is achieved by means of a complex fusion of fascio-ligamentous structures, where the iliopubic tract, the medial condensations of the transversalis fascia and the lacunar ligament are the most important.
\end{abstract}

\section{Keywords}

Femoral Hernia, Femoral Canal, Transversalis Fascia, Groin Anatomy

\section{Introduction}

The anatomic region through which most inguinal hernias emerge is the myopectineal orifice, which is divided by the inguinal ligament into a superior area and an inferior area [1]. The inferior area is the site where femoral 
hernias appear. But this confined area is overcrowded by various anatomical structures which are intricately inter-related. This is reflected by the wide range of anatomic interpretations of their position and role in shaping the groin where femoral hernia appears.

Despite with the contributions by many authors who have refined our knowledge, surgeons prefer using simplified anatomical parameters: the annulus femoralis is bordered anteriorly by the inguinal ligament, medially by the lacunar ligament, posteriorly by the pectineal (Cooper's) ligament and laterally by the femoral vein [2]. As noted by R. Bendavid — nothing that touches upon femoral hernia can ever be simple [3].

But the greatest mystery surrounds the term "femoral canal". Here the opportunity presents two options: its presence and conversely, its absence. The presence of femoral canal is considered by some to be a normal anatomical fact [4]-[6], while others do not support this assertion [1]. Several studies have been carried out in this direction [7]-[10]. However, with the introduction of laparoscopic inguinal hernia operations, the following question is raised: if there was indeed a femoral canal, should not this then, lead to the appearance of subcutaneous emphysema of the thigh — at the site of the operation? This is valid mainly for the extraperitoneal repairs, where this kind of emphysema has not been reported [11] [12].

The circumstances mentioned above serve as a preface to our studies on the topic to be shared. During cadaver dissections and groin hernia repairs, examinations were carried out on the anatomic structures and their relationship to the presence of a femoral hernia [13].

\section{Material and Methods}

The inguinal and the lacunar ligaments, the iliopubic tract and the transversalis fascia were examined, and the possibilities for the existence of the femoral canal considered. The distance between the pubic tubercle and the medial margin of the femoral vein as well as the distance between the inguinal and the Cooper's ligaments were measured. The study was done between 1978 and 1984 in the departments of surgery and pathological anatomy at the former Higher Medical Institute, today Medical University, in Pleven [13].

1) Over 100 fresh cadavers were studied, covering an equal number of men and women; none had a hernia or a previous regional injury. The following techniques were used:

a) Trans-inguinal incision with additional medial perpendicular extension over the base of the thigh.

b) Preperitoneal approach with removed abdominal content.

c) Preperitoneal approach with preserved abdominal content.

In the course of the dissection the anatomical structures under investigation were measured and the results were documented through text, sketches and photographs.

2) Studies were done on 47 patients with femoral hernias - 41 by inguinal, 5 by preperitoneal (two with bilateral localizations), and 1 by trans-abdominal approach. A control group consisted of 10 male patients with direct hernias. The results were recorded in a manner similar to the cadaver study, with the exception of photography.

Alternative and variance analyses were used to process the results with a confidence limit $t=0.95$. In the variance analysis the level of significance was estimated according to the null hypothesis using the t-criterion.

\section{Results}

1) The lacunar ligament

The dissection studies demonstrated that the lacunar ligament is present in $74 \%$ of men and $50 \%$ of women. It was a significant structure in $50 \%$ of men and $24 \%$ of women. In these cases, the ligament measured from 1 to $1.5 \mathrm{~mm}$ in thickness and stretched laterally between 2 and $2.5 \mathrm{~cm}$. Its lateral edge passes in the transversalis fascia which covers it with an interposition of fine fatty tissue, which is enlarged in overweight patients (Figure 1). When the lacunar ligament was not found, the inguinal ligament, the pectineal fascia and fascia lata replaced it with good consolidation of the area.

In patients who underwent surgery the lacunar ligament was found with the same frequency as in the dissection studies. It was well expressed in $25 \%$ of male and $30 \%$ of female patients, with statistical significance only for women.

2) The iliopubic tract

Crossing the external iliac vessels behind the inguinal ligament, the iliopubic tract was medially fused with the transversalis fascia. It received fibers from the inguinal ligament, with which it was well integrated. We 
found this in $60 \%$ of dissections (Figure 2). Medially, the iliopubic tract gave fibres, which were directed into the lacunar ligament. In $40 \%( \pm 9.6)$ it was merged with the transversalis and the pectineal fascia.

In about half of the patients who underwent surgery for femoral hernias the iliopubic tract was not well represented.

3) Dissection studies and transversalis fascia

Many surprising relationships were found distally, in the distal parts of the transversalis fascia and medially from the femoral vein. In $65 \%( \pm 9)$ of cases it had received fibers from the transversus abdominis muscle. Distally it had fused with the iliopubic tract and pectineal fascia with angular splitting. We were able to identify four variations in its distal fusions - with the iliopubic tract of the anterior leaf, with Cooper's ligament and with the pectineal fascia on its posterior aspect. The following variations were seen most frequently: type I in $17 \%$, type II in 25\%, type III in 20\% and type IV in 20\% (Figures 3-6).

It was found that the transversalis fascia did not pass distally into the lymphatic lacuna. Medially this fascia did not reach the lacunar ligament, but was rather positioned above it forming the vascular sheath laterally. Here the fascia participates in the formation of a fossa, which varies in width and depth. Beside the fascia transversalis, laterally the fascia iliaca and fascia lata participate in the formation of the vascular sheath in a similar fashion.

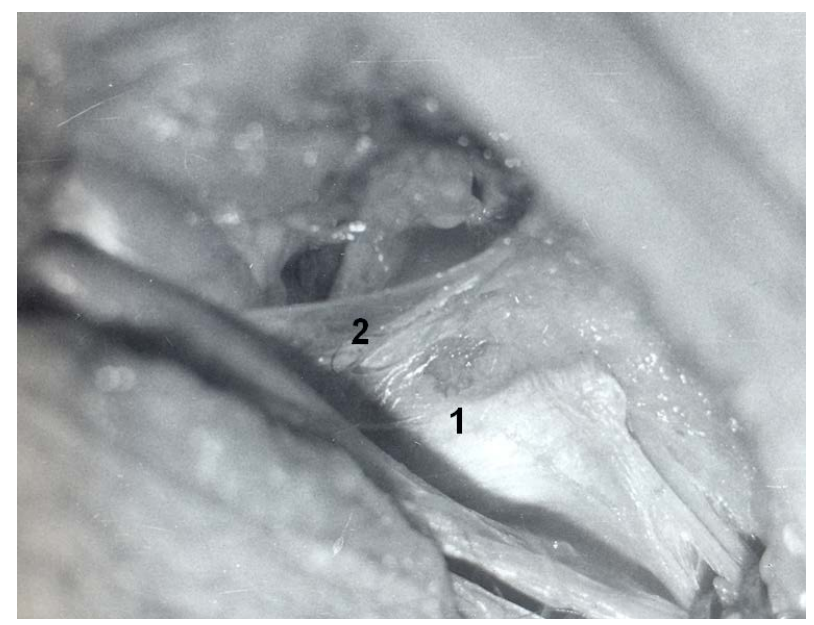

Figure 1. Right side: 1-lacunar ligament; 2-transversalis fascia.

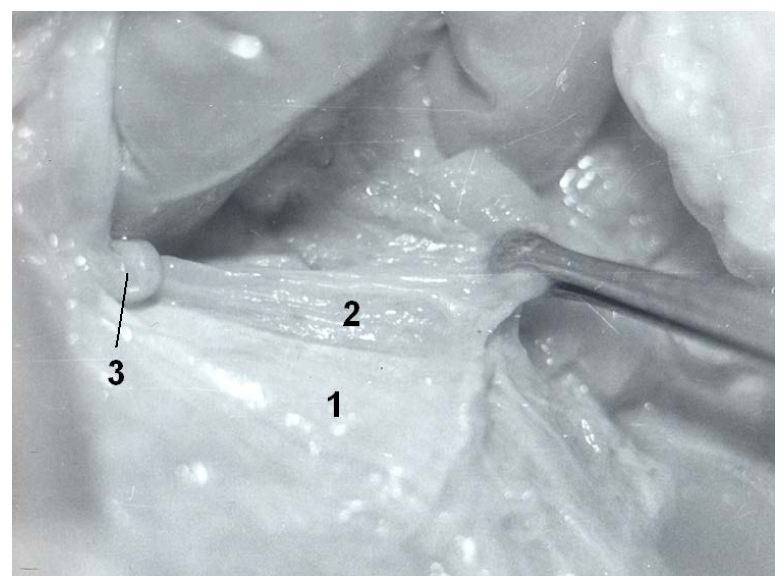

Figure 2. Right groin (anterior view): 1-external obl. aponeurosis leaf; 2-iliopubic tract; 3-Rosenmuller-Cloquet nodule: the inguinal ligament is removed, and iliopubic tract together with $\mathrm{f}$. transversalis and $\mathrm{f}$. pectinea are mobilized from the pecten ossis pubis and grasped by pincette. 


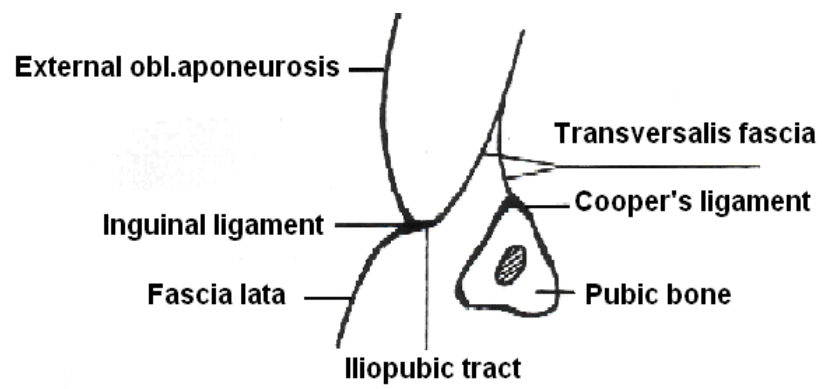

Figure 3. Type 1 transversalis fascia distal fusion (17\%).

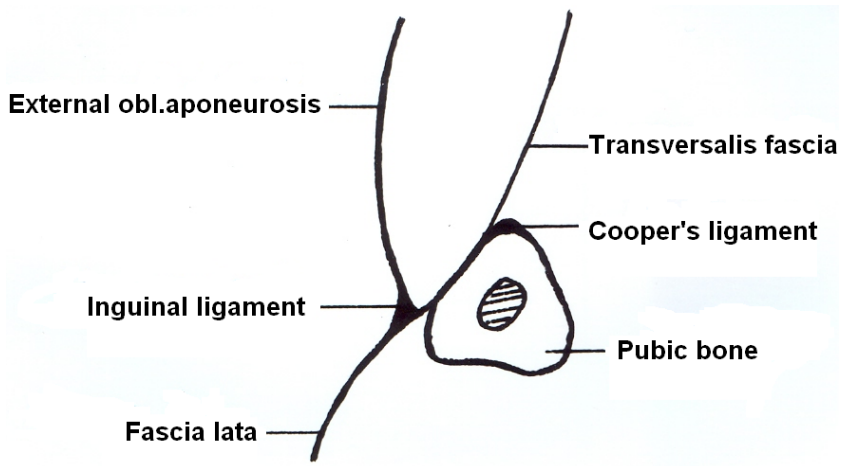

Figure 4. Type 2 transversalis fascia distal fusion (25\%).

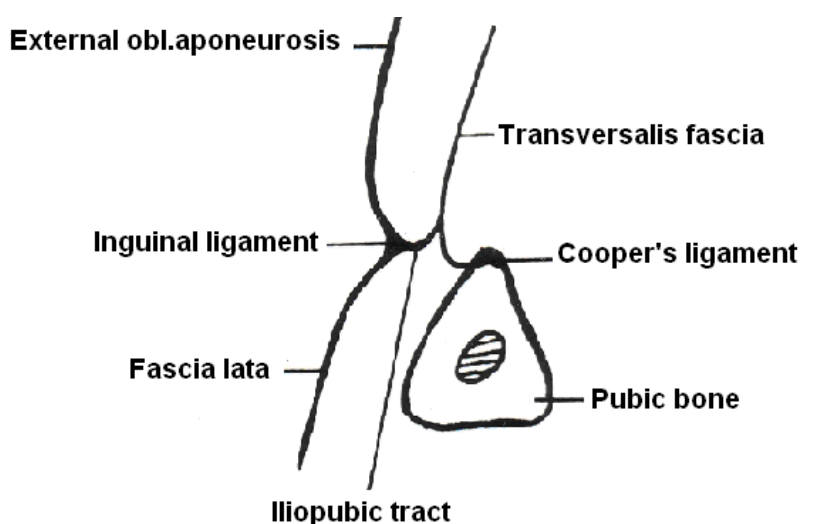

Figure 5. Type 3 transversalis fascia distal fusion (20\%).

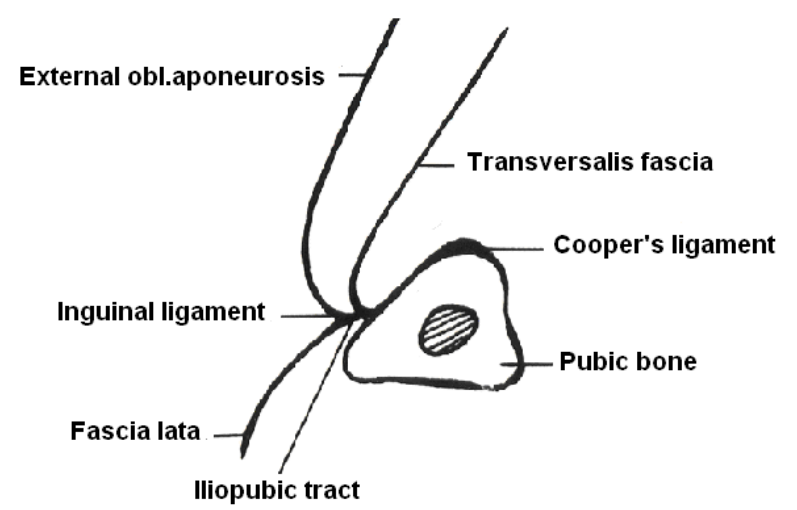

Figure 6. Type 4 transversalis fascia distal fusion (20\%). 
4) Distance between the Cooper's and inguinal ligaments

a) In dissections studies the distance between the two ligaments was $2.2 \mathrm{~cm}$ for men and $1.9 \mathrm{~cm}$ for women (Diagram 1).

b) In men who underwent surgery the measured distance was $2.1 \mathrm{~cm}$, while in women it was $2.5 \mathrm{~cm}$ (Diagram 2). In ten male patients with direct inguinal hernias the average distance between the two ligaments was comparable (Diagram 3).

c) When comparing the results of the two types of studies we did not find any differences in men (Diagram 4), but in women with femoral hernia there was a statistical difference of $0.6 \mathrm{~cm}$ (Diagram 5).

5) The distance between the medial wall of the femoral vein and the pubic tubercle

a) In dissections there was no difference between men and women (Diagram 6).

b) In patients who underwent surgery the average distance was $3.2 \mathrm{~cm}$ in men and $3.4 \mathrm{~cm}$ in women with possibilities for individual variations (Diagram 7).

c) The comparison of the results between the above mentioned groups points to the lack of significant differences between males but a difference in the measurements in females (Diagram 8, Diagram 9).

6) Femoral fossa

While studying the distal transversalis fascia we found that medial to the femoral vein it forms a fossa that varies in measurement (Figure 7). This corresponds with the above 3 and 4 types of distal fusion. Its boundaries were: anteriorly the iliopubic tract and occasionally fascia lata, medially the iliopubic tract and in some cases the lacunar ligament, laterally its own femoral sheath and the deeper layers of fascia lata, and posteriorly the fascia pectinea. In the dissections it was found in 32\% of male and 30\% of female subjects (Table 1).

In patients at surgery, such a fossa was found in 3 men and 9 women (Table 2). It existed, despite the pres-

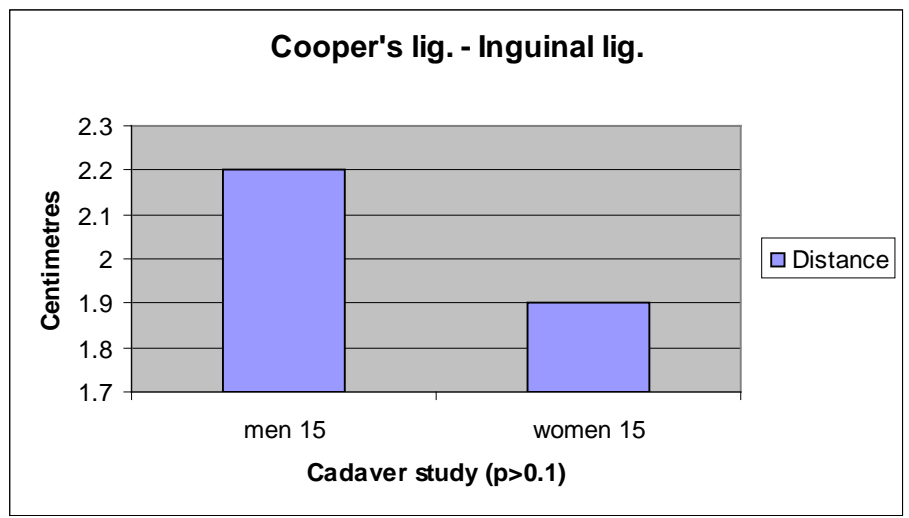

Diagram 1. Average distance between the Cooper's and inguinal ligament in dissections studies.

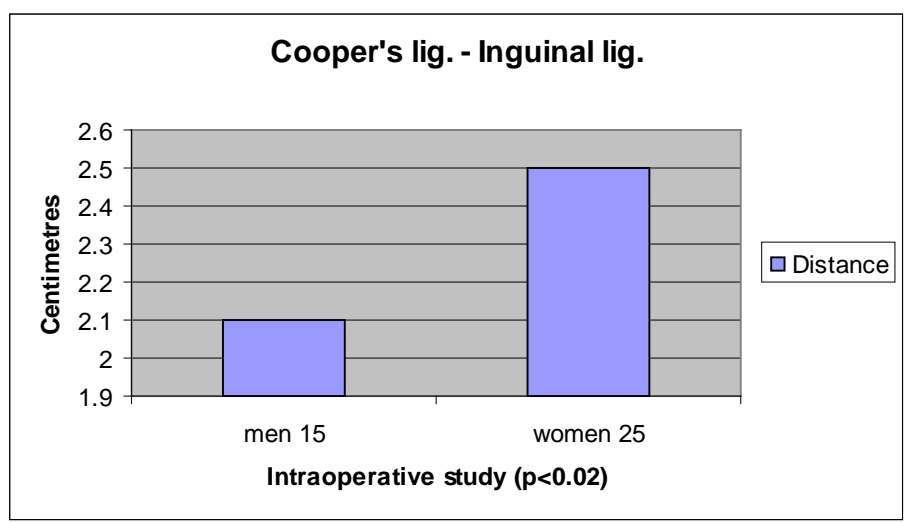

Diagram 2. Average distance between the Cooper's and inguinal ligament in patients who underwent surgery. 


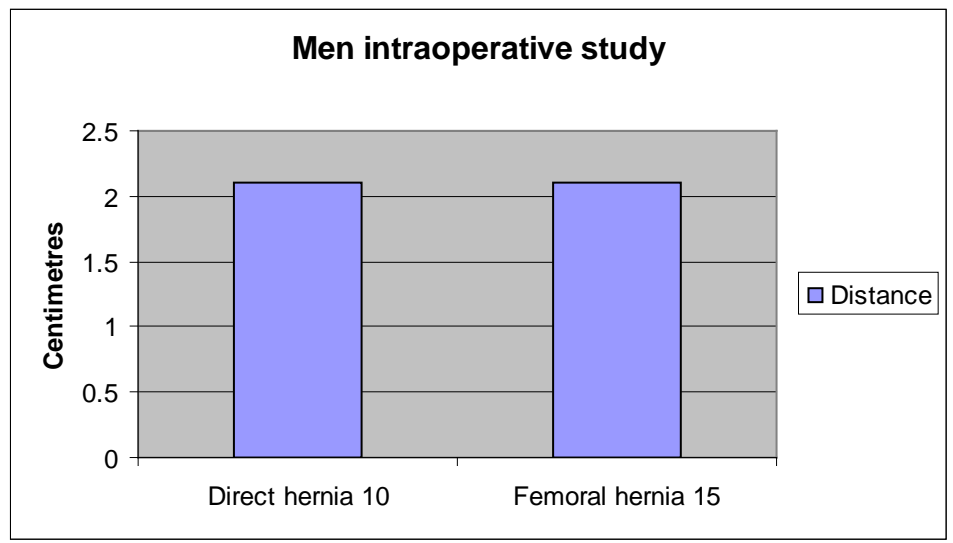

Diagram 3. Average distance between the Cooper’s and inguinal ligament.

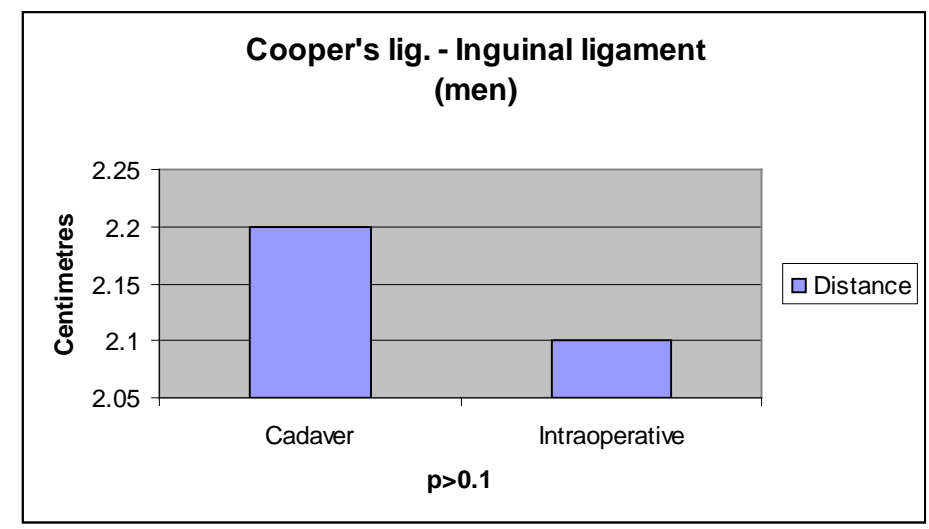

Diagram 4. Comparison of the average distance between the cadaver and intraoperative studies in men.

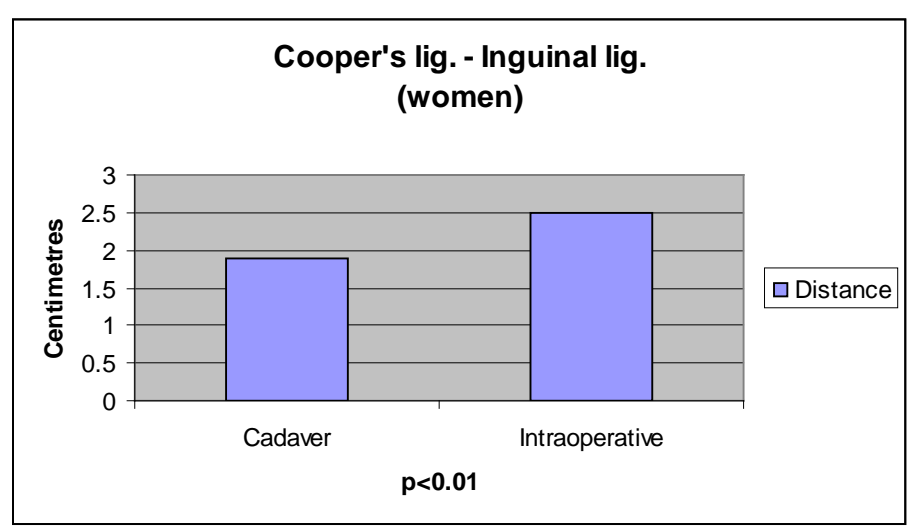

Diagram 5. Comparison of the average distance between the cadaver and intraoperative studies in women.

Table 1. Dimensions of the preperitoneal femoral fossa (cadaver study).

\begin{tabular}{ccc}
\hline Parameter & Men & Women \\
\hline Diameter at base $(\mathrm{cm})$ & $1.7 \pm 0.18$ & $0.7 \pm 0.14$ \\
Depth $(\mathrm{cm})$ & $1.3 \pm 0.28$ & $1.4 \pm 0.22$ \\
\hline
\end{tabular}


Table 2. Dimensions of the preperitoneal femoral fossa (intraoperative study).

\begin{tabular}{ccc}
\hline Parameter & Men & Women \\
\hline Diameter at base $(\mathrm{cm})$ & $1.5 \pm 0.01$ & $2.5 \pm 0.07$ \\
Depth $(\mathrm{cm})$ & $1.0 \pm 0.04$ & $1.4 \pm 0.07$ \\
\hline
\end{tabular}

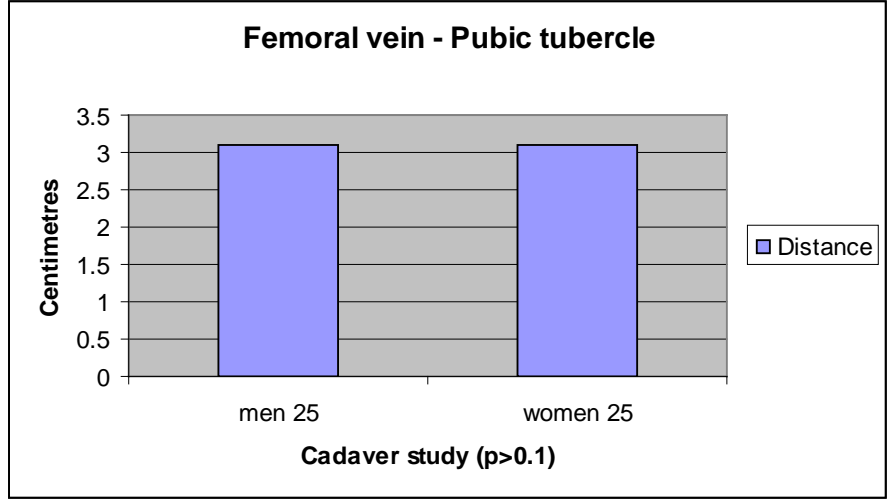

Diagram 6. Average distance between medial wall of femoral vein and pubic tubercle (cadaver study).

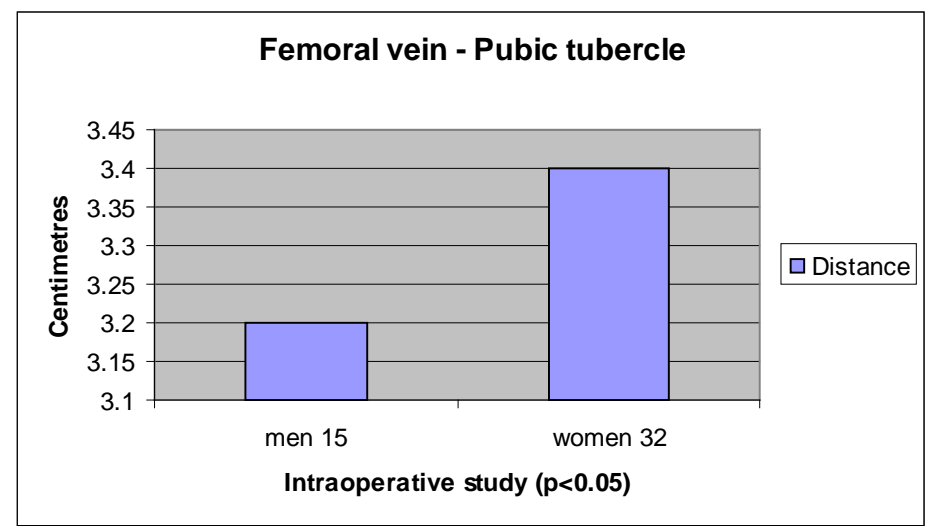

Diagram 7. Average distance between medial wall of femoral vein and pubic tubercle in patients (intraoperative study).

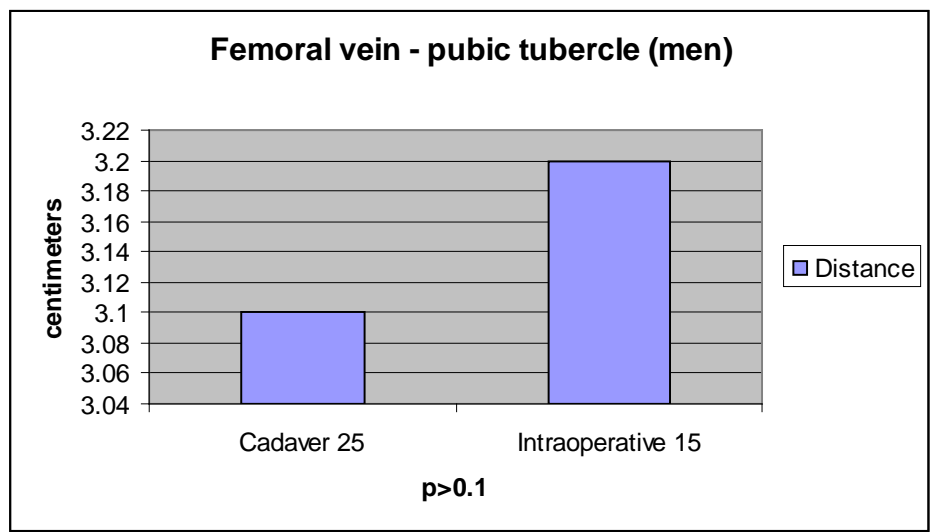

Diagram 8. Comparison of the average distance between the cadaver and intraoperative studies in men. 


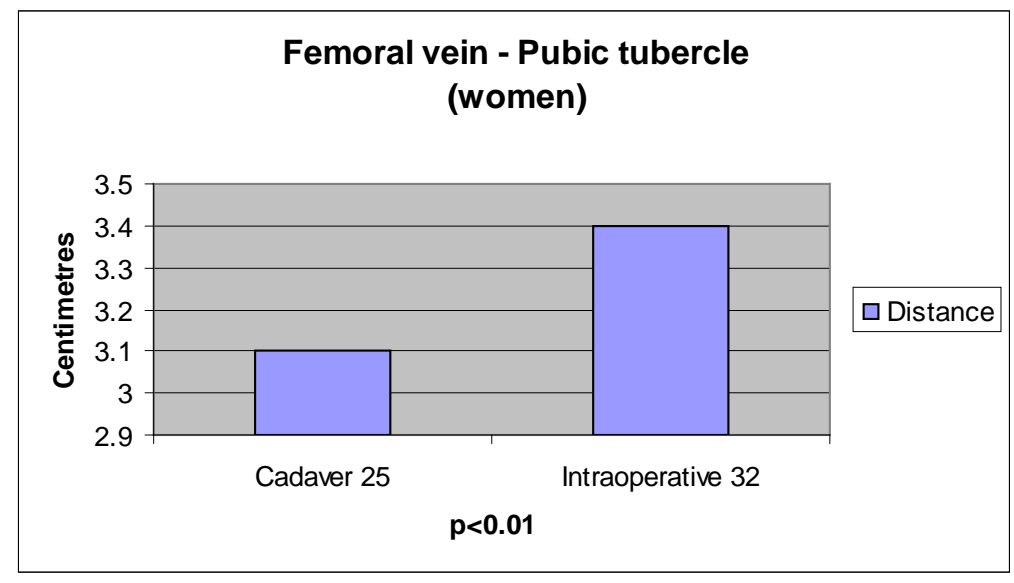

Diagram 9. Comparison of the average distance between the cadaver and intraoperative studies in women.

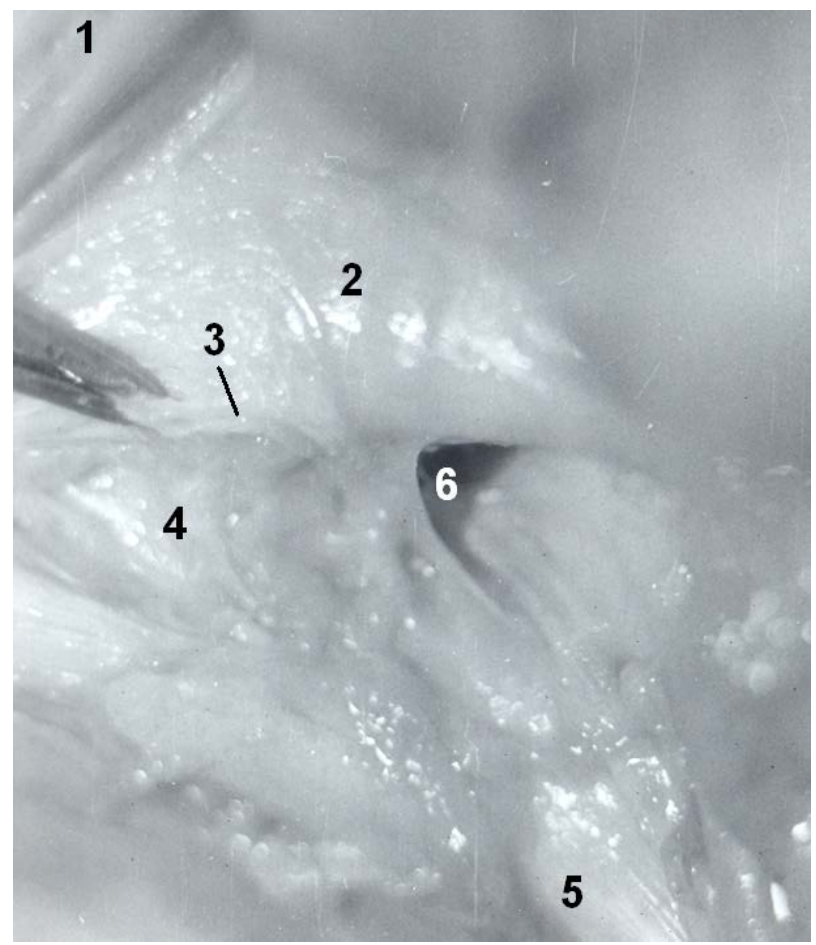

Figure 7. Right inguinal area (preperitoneal view): 1 -rectus abd.muscle; 2-transversus abd.muscle; 3-incision of the transversalis fascia; 4-lacunar ligament; 5-Cooper's ligament; 6 - well expressed preperitoneal femoral fossa.

ence of femoral hernia, lateral to the hernial sac adjacent and medial to the vascular sheath. This anatomic entity was described as preperitoneal femoral fossa (foveola femoralis preperitonealis) (Figure 8) [13].

7) Presence of femoral canal in subjects without femoral hernia

In cadaver studies it was established that in $56 \%$ of cases the iliopubic tract, the transversalis fascia and the lacunar ligament, when present, together with fascia pectinea blocking the lymphatic lacuna (Figure 9). In 45\% the above mentioned lacuna was protected distally by the iliopubic tract and fascia lata. In those, a femoral canal was not found.

8) Femoral hernia canal (Figure 10) 


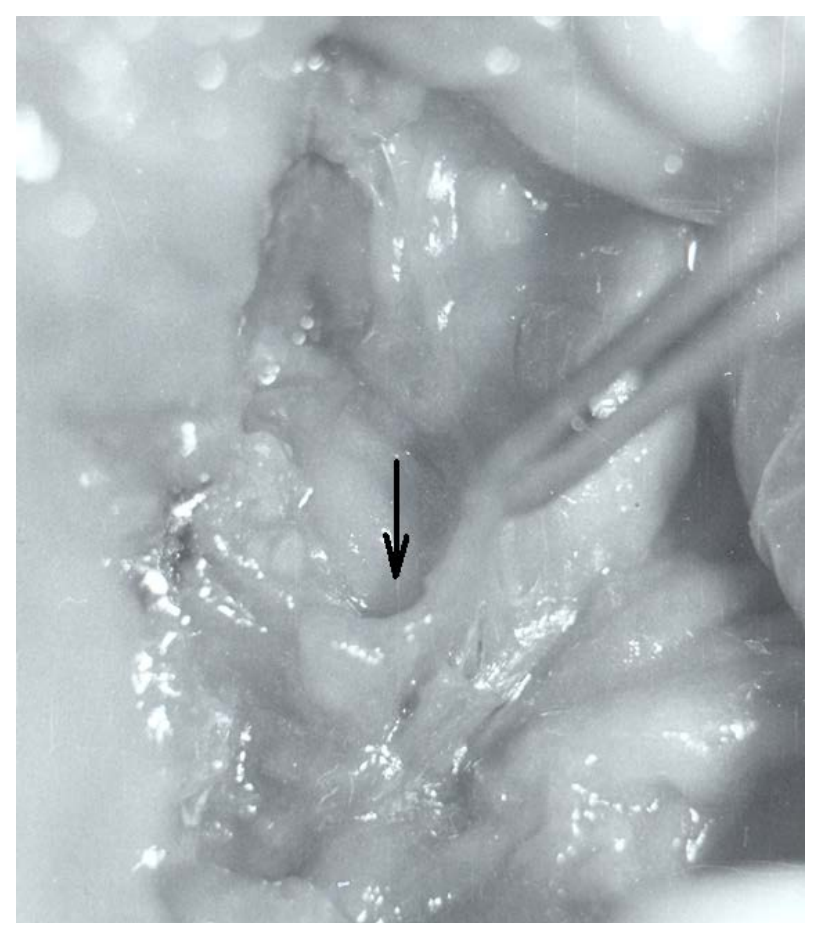

Figure 8. Right femoral area (anterior view): pick up with a pincette the obliterated from below preperitoneal femoral fossa (arrow).

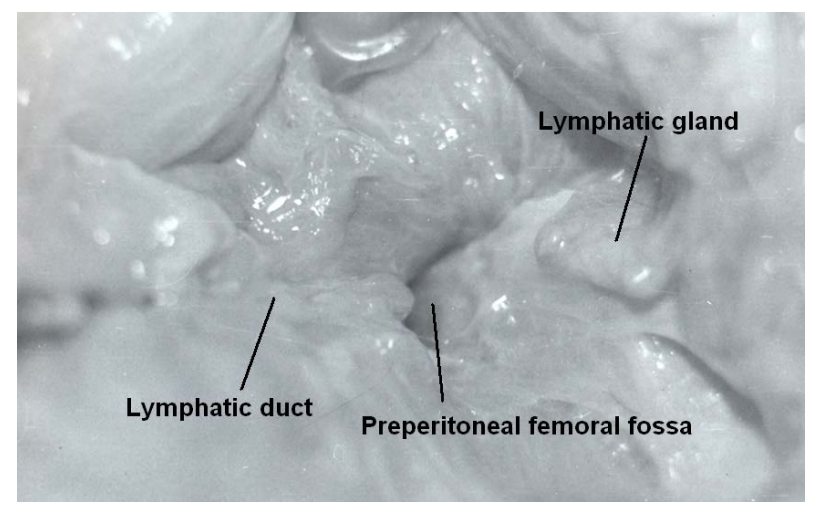

Figure 9. Left preperitoneal anterior view.

In the course of surgeries, we established the presence of three zones that are rigid and have characteristic narrowings. Their definition depends on their position: proximal, intermediate, and distal [13].

The proximal zone was formed anteriorly and medially by the transversalis fascia and a layer of the transversus abdominis, posteriorly by Cooper's ligament, laterally by the transversalis fascia with fibers from the interparietal fascia and the fascial part of transversus abdominis. This, in fact, is the entrance of the femoral hernia canal.

The intermediate zone was formed anteriorly by the inguinal ligament and posteriorly, the iliopubic tract, medially by the lacunar ligament (when the latter is not present by the iliopubic tract), dorsally by the pectineal fascia, and laterally by trasversalis fascia and additional fibers which formed as a result of the hernia.

The distal narrowing had the following borders: anteriorly the fibers from the inguinal ligament and fascia lata, medially the fascia lata, and laterally and dorsally the deep layers of fascia lata. This ring formed the femoral hernia exit site.

The length of the described canal varied between 2.5 and $3 \mathrm{~cm}$; it is not a cylinder with perpendicular bases. 


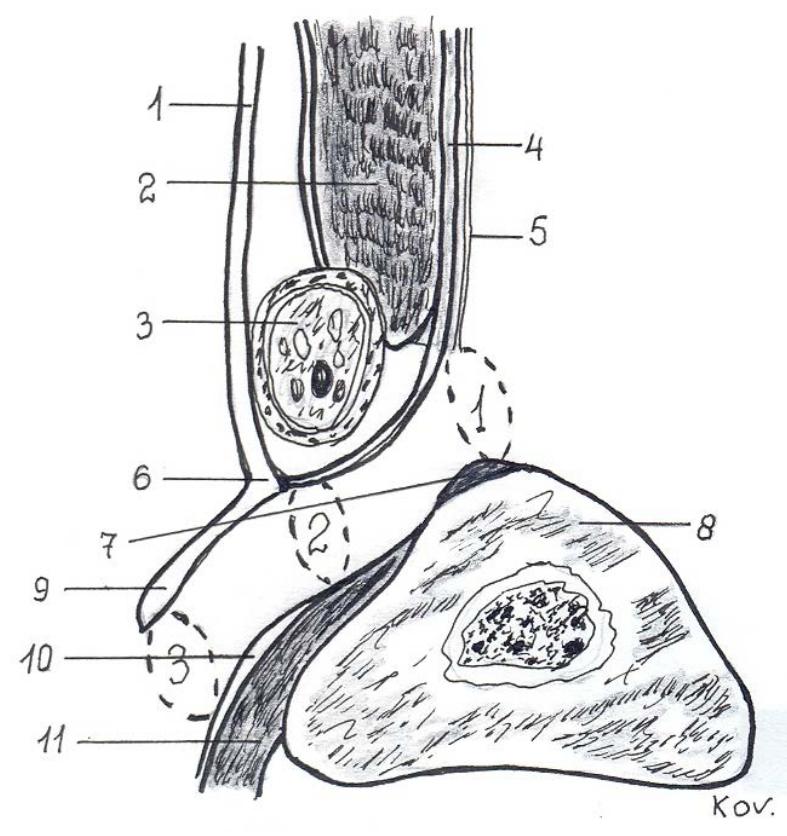

Figure 10. Femoral hernia canal with its rigid of narrowings: 1-the proximal; 2-the middle; 3-the distal. 1-external oblique aponeurosis; 2-internal oblique muscle; 3-spermatic cord; 4-transversus abd.muscle; 5-transversalis facsia; 6-inguinal ligament; 7-Cooper's ligament; 8-pubic bone; 9-fascia lata; 10 - pectineal fascia, 11 -pectineus muscle.

The latter-the entrance and exit rings—are positioned at an oblique angle in relation to the canal's axis. The distance between the intermediate narrowing and the exit varies between 1.5 and $2 \mathrm{~cm}$. In upright position the femoral hernia canal ran in an oblique angle, with occasional frontal convexity.

Intra-operatively we found that the hernia sac was fixed in the proximal ring in $19.1 \%$ (9 patients), in the middle in $59.5 \%$ (28 patients) and in the distal in $21.2 \%$ (10 patients) of patients.

\section{Discussion}

W.J. Lytle has described in great detail the lacunar ligament [8]. In comparison with the control group, it varies considerably amongst women with femoral hernias. Its absence was compensated by the solid condensation of the inguinal ligament, pectineal fascia and fascia lata. As a layer of the transversus abdominis, the iliopubic tract crosses before the external ilio-femoral vessels from the iliopectineal arch to superior pubic ramus [9]. The latter gives grounds to Bendavid to accept that the transversus abdominis muscle provides fibers to this ligament [14]. Medially the iliopubic tract plays an important role in strengthening the femoral area (Figure 11). In patients with operations from femoral hernias, the iliopubic tract varied significantly and in half of the patients it was not particularly substantial. It is in close relation to the transversalis fascia which receives fibers from the aponeurosis of the transversus abdominis muscle as well—a fact noted by other authors [15].

A new moment in the anatomy of the medial femoral area is the distal splitting of transversalis fascia anteriorly to the iliopubic tract, and posteriorly to Cooper's ligament and pectineal fascia. This could be identified as the separation of bilaminar transversalis fascia as described by A. Cooper, J. Cleland et al. and R. Read [16]-[18]. But what really drew our attention was that above the bifurcation zone transversalis fascia appeared homogenous; its two layers are practically indistinguishable. Skandalakis et al. describe a fusion of transversus abdominis aponeurosis with the transversalis fascia at the region of the posterior wall of the inguinal canal [19]. Pierpont $e t$ al. found muscular fibers of the transversus abdominis muscle which are fixed at the transversalis fascia and nearly inseparable [20]. These observations are similar to those done of other authors [14].

This latter finding is especially important for our study, and more precise to the fact that the transervsalis fas- 
cia participates in the formation of the preperitoneal femoral fossa. This corresponds with the presence of two of its variations of distal bifurcation. Laterally transversalis fascia together with the iliac fascia and fascia lata composes the femoral vascular sheath. Since the preperitoneal femoral fossa was present in cases of femoral hernia, this confirms that it is an anatomical entity which differs from the site where the hernia is found (Figure 12). Rodriguez et al. described a structure which they identified as femoral canal, yet it could be the femoral fossa [10].

The descriptions of the anatomy of the subinguinal groin area are multifaceted and by no means uniform. C.B. McVay thought that the space in the lacuna vasorum between the medial wall of the femoral vascular sheath and the femoral vein is the femoral canal. He identified this border at the aponeurotic insertion of the transversus abdominis and the lacunar ligament. This ligament builds the ring only when the latter is distended [21].

W.J. Lytle is certain that the femoral canal exists [8]. He followed McVay's ideas about the formation of the femoral vascular sheath and the contribution of the lacunar ligament. He further describes the femoral trough which houses the femoral canal on each side and which results from the forward position of the flat muscles of the anterior abdominal wall which pass down well in front of Cooper's ligament and turn $1-1.5 \mathrm{~cm}$ back further down to join the pectineus muscle fascia. The trough measures about $12 \mathrm{~cm}$ in length and extends transversely between the external iliac veins inclining slightly downward towards the symphysis pubis [8]. According to Lytle the femoral canal, lined by transversalis fascia, occupies the lateral part of the femoral trough on each side. It is rightly named a canal: it lies transversely and is not a vertically placed funnel. The canal measures from 3 to $4 \mathrm{~cm}$ in transverse length and from 1 to $1.5 \mathrm{~cm}$ in depth. It opens into the abdominal cavity at the femoral ring whose boundaries are: the external iliac vein lateral; Cooper's ligament behind; the posterior inguinal wall in front and medial, a low barrier of fascial and tendinous fibres passing back from the rectus muscle to the pelvic brim. The medial boundary of the ring however, is not the curved edge of the lacunar ligament, which lies further down at the upper end of the femoral sheath, at the level of the inguinal ligament [8].

In our description of the femoral hernia canal, each of the three zones corresponds to some of the descriptions above [13]. Thus Lytle identifies the first and second narrowing [8]; A. Temiz et al.- the second one [2]. What is the clinical importance of recognizing these three rigid zones of the passage of the femoral hernia canal? They are the potential sites for incarceration of femoral hernias!

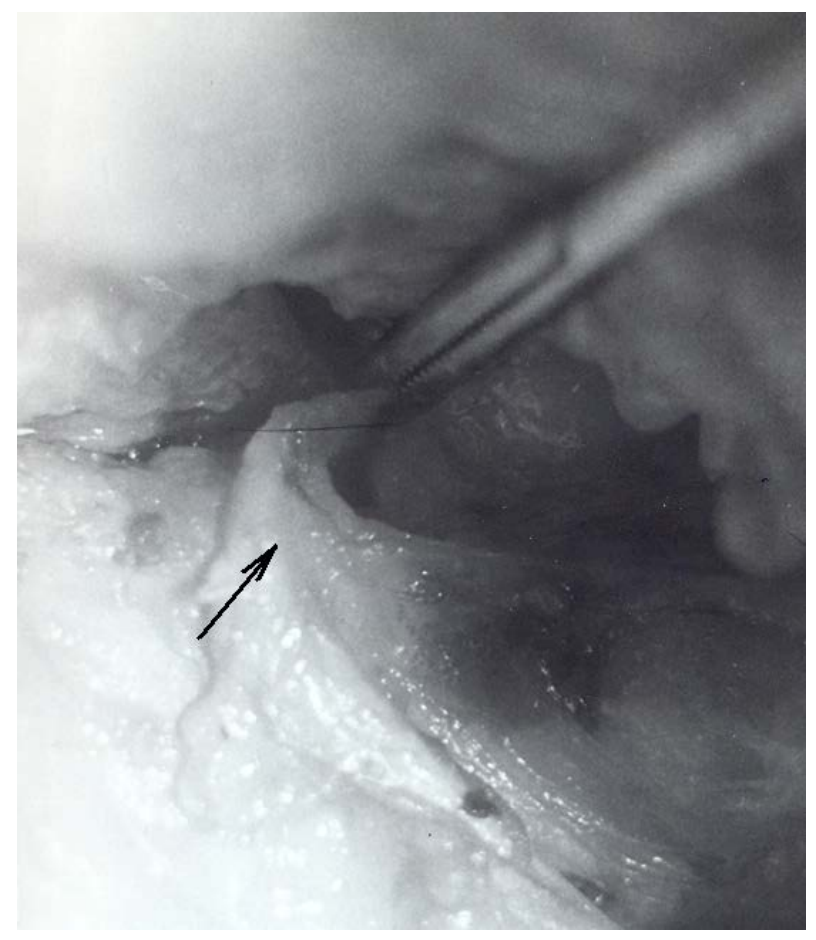

Figure 11. Left groin (anterior view): a demonstration of its sound fibrous closure (arrow) with participation of the iliopubic tract. 


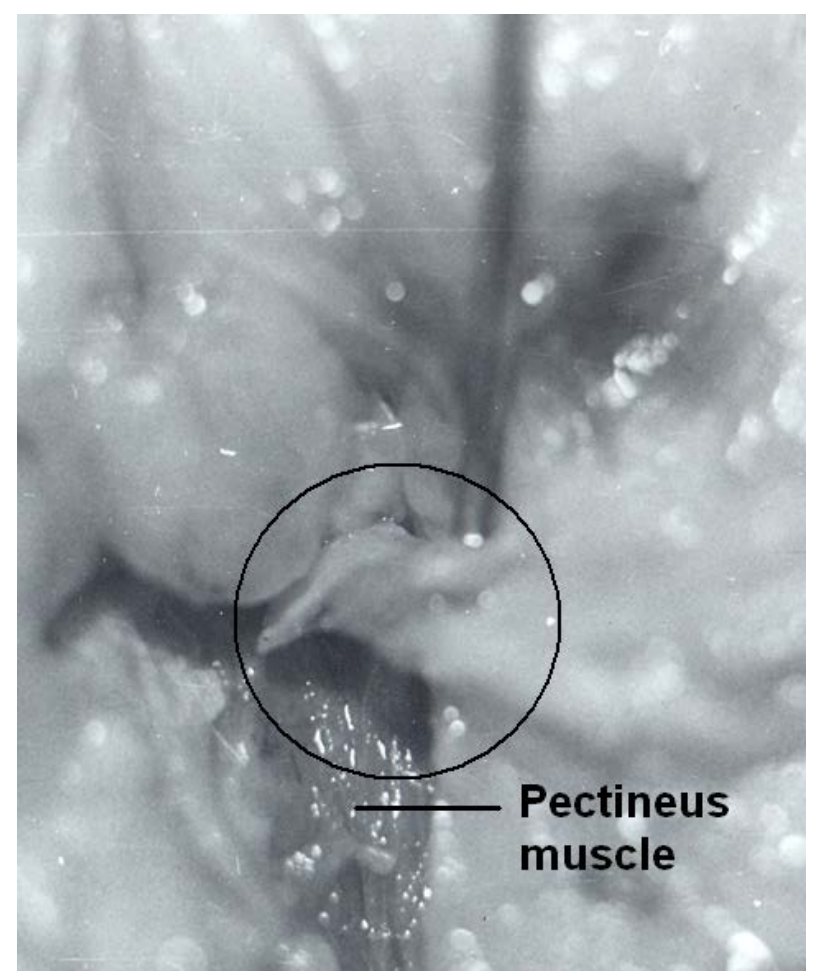

Figure 12. Left groin (anterior view): the pectineal fascia is dissected together with structures of the femoral fossa (encircled) with introduced in the fossa probe.

When these happen in the first and second narrowing the common swelling in the sub-inguinal area may not be present even though it is considered to be a typical symptom of femoral hernia. This could explain another fact: in different individuals the preperitoneal space contains varying amounts of fat; when this fat is stuck in the upper two rings it could turn into a plug and femoral hernia would not become apparent. This could explain why laparoscopic surgeons report higher frequency of femoral hernias on the order of $11 \%$ in comparison to traditional statistics (2\% in males and $7 \%$ in females) [3]. The clarification for this is because of the existence of the preperitoneal fossa with fat which is not a femoral hernia. $\mathrm{R}$. Bendavid is correct when he notes that the femoral pocket should not be disturbed unless a femoral hernia is clinically suspected [3].

How can one interpret the data from the comparative metrical studies of the distance between Cooper's ligament and the inguinal ligament on the one hand and between the medial wall of the femoral vein and pubic tubercle on the other? The results clearly indicate that in women with femoral hernias these distances are much larger. This shows a parallel with the role of Hessert's triangle [22] at the posterior wall of the inguinal canal in the development of inguinal hernias with the importance of these spaces for the formation of femoral hernias. This explains why femoral hernias occur more frequently in women rather than men. Furthermore in women with femoral hernias these parameters are better expressed in comparison with those in the control group.

Is there anything constant about the femoral canal? Plenty of speculations exist since the early publications on the subject by J. Cloquet [23]. It is interesting that already T.P. Teale has described three areas, of which the lateral two areas are occupied by the femoral artery and vein. The third, the pubic compartment is occupied by the lymphatic vessels of the lower limb; it is into the pubic compartment of the femoral sheath that a femoral hernia descends [24]. Nowadays we can accept that there are three lacunae underneath the iliopubic tract: muscular, vascular, and lymphatic [1] [25]. The lymphatic lacuna presents complicated anatomical relationships and is the place where femoral hernias occur. R. Bendavid has accepted the presence of a blind pocket, lined with the transverse fascia, whose lower end extends to the level of the fossa ovalis [3]. In reality, this is the described preperitoneal femoral fossa in this study. So, this means that without hernia, femoral canal does not exist.

In the lower infra-inguinal space in which femoral hernias could develop, its integrity is realized through the complex fusion of fascio-ligamentous structures, where the iliopubic tract, the medial condensation of the trans- 


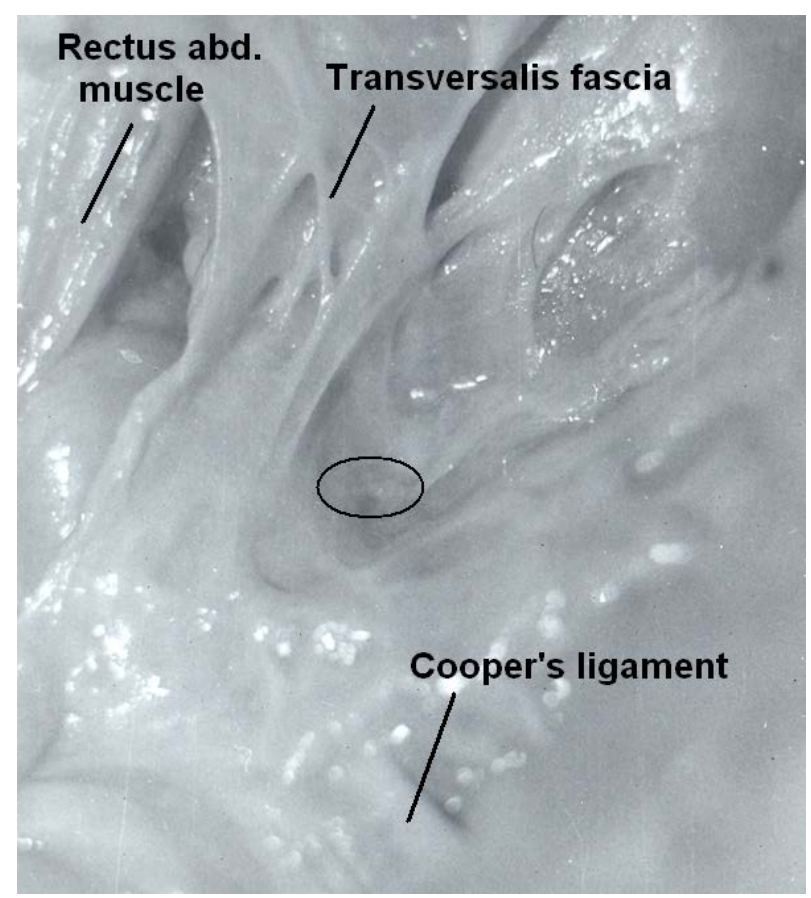

Figure 13. Right inguinal preperitoneal view with absent preperitoneal femoral fossa (ellipse).

versalis fascia and the lacunar ligament are most important (Figure 13). Their solidity is biologically variable [26], but anatomically determined. In the context of the latter the unified and clear presentation of the anatomy in this area will aid clinical practice. The use of new technologies of hernia repair helps to update our traditional views regarding femoral hernias and their anatomic characteristics.

\section{References}

[1] Fruchaud, H. (1956) The Surgical Anatomy of Hernias of the Groin. G. Doin, Paris.

[2] Temiz, A., Akcora, B., Temiz, M. and Canbolant, E. (2008) A Rare and Frequently Unrecognised Pathology in Children: Femoral Hernia. Hernia, 12, 553-556. http://dx.doi.org/10.1007/s10029-008-0360-6

[3] Bendavid, R. (2002) Femoral Pseudo-Hernias. Hernia, 11, 91-112.

[4] Chapman, W.H.H. (1991) Femoral Hernia in Children: An Infrequent Problem Revisited. Military Medicine, 156, 631663.

[5] Al-Shanafey, S. and Giacomantonio, M. (1999) Femoral Hernia in Children. Journal of Pediatric Surgery, 34, 11041106. http://dx.doi.org/10.1016/S0022-3468(99)90576-6

[6] Alimoglu, O., Kaya, B., Okan, I., Dasiran, F., Guzey, D., Bas, G. and Sahin, M. (2006) Femoral Hernia: A Review of 83 Cases. Hernia, 10, 70-73. http://dx.doi.org/10.1007/s10029-005-0045-3

[7] McVay, C.B. and Savage, L.E. (1961) Etiology of Femoral Hernia. Annals of Surgery, 154, 25-32. http://dx.doi.org/10.1097/00000658-196112000-00005

[8] Lytle, W.J. (1979) Inguinal Anatomy. Journal of Anatomy, 128, 581-594.

[9] Skandalakis, J.E., Colborn, G.L., Androulakis, J.A., Skandalakis, L.J. and Pemberton, L.B. (1993) Embryologic and Anatomic Basis of Inguinal Herniorrhaphy. Surgical Clinics of North America, 73, 799-836.

[10] Rodrigues Jr., A.J., Rodrigues, C.J., Plopper, C. and Terra, R.M. (2000) Do the Dimensions of the Femoral Canal Plays Role in the Genesis of Femoral Hernia? Hernia, 4, 141-143. http://dx.doi.org/10.1007/BF01230594

[11] Ferzli, G.S., Kiel, T., Hurwitz, J.B., Davidson, P., Piperno, B., Fiorillo, M.A., Hayek, N.E., Riina, L.L. and Sayad, P. (1997) Pneumothorax as a Complication of Laparoscopic Inguinal Hernia Repair. Surgical Endoscopy, 11, 152-153. http://dx.doi.org/10.1007/s004649900320

[12] Ramia, J.M., Pardo, R., Cubo, T., Padilla, D. and Hernandez-Calvo, J. (1999) Pneumomediastinum as a Complication of Extraperitoneal Laparoscopic Inguinal Hernia Repair. JSLS, 3, 233-234. 
[13] Kovachev, L. (1985) Anatomic Preconditions for Femoral Hernia Appearance and Its Surgical Treatment. Ph.D. Dissertation, Medical University, Pleven. (in Bulgarian)

[14] Bendavid, R. and Howart, D. (2000) Transversalis Fascia Rediscovered. Surgical Clinics of North America, 80, 25-33. http://dx.doi.org/10.1016/S0039-6109(05)70395-9

[15] Anson, B.J., Morgan, E.H. and McVay, C.B. (1960) Surgical Anatomy of the Inguinal Region Based upon a Study of 500 Body-Halves. Surgery, Gynecology Obstetrics, 111, 707-725.

[16] Cooper, A.P. (1804) The Anatomy and Surgical Treatment of Inguinal and Congenital Hernia. Longman, London.

[17] Cleland, J., Mackay, J.Y. and Young, B.J. (1989) The Relations of the Aponeurosis of the Transverses and Internal Oblique Muscles to the Deep Epigastric Artery and to the Inguinal Canal. In: Memoirs and Memoranda in Anatomy, Vol. 1, Williams and Norgate, London.

[18] Read, R.C. (1992) Cooper’s Posterior Lamina of Transversalis Fascia. Surgery, Gynecology \& Obstetrics, 174, 426434.

[19] Skandalakis, J.E., Gray, S.W., Skandalakis, L.J., Colborn, G.L. and Pemberton, L.B. (1989) Surgical Anatomy of the Inguinal Area. World Journal of Surgery, 13, 490-498. http://dx.doi.org/10.1007/BF01658861

[20] Pierpont, R.Z., Grigoleit, A.W. and Finegan, M.K. (1969) The Transversalis Fascia: A Practical Analysis of an Enigma. American Surgeon, 35, 737-740.

[21] McVay, C.B. (1974) The Anatomic Basis for Inguinal and Femoral Hernioplasty. Surgery, Gynecology \& Obstetrics, 139, 931-945.

[22] Abdala, R.Z. and Mittelstaedt, W.E. (2001) The Importance of the Size of Hessert's Triangle in the Etiology of Inguinal Hernia. Hernia, 5, 119-223. http://dx.doi.org/10.1007/s100290100024

[23] Cloquet, J. (1817) Recherches anatomiques sur les hernies de l'abdomen. Librairie Mequignon-Marvis, Paris, 67-68.

[24] Teale, T.P. (1846) A Practical Treatise on Abdominal Hernias. Longman, Brown, Green and Longman, London, 301305.

[25] Kovachev, L. (2004) Groin Physiology. In: Kovachev, L., Ed., Groin Hernia, Eurasia Abagar, Pleven, 42-57. (in Bulgarian)

[26] Franz, M.G. (2006) The Biology of Hernias and the Abdominal Wall. Hernia, 10, 462-471. http://dx.doi.org/10.1007/s10029-006-0144-9 
Scientific Research Publishing (SCIRP) is one of the largest Open Access journal publishers. It is currently publishing more than 200 open access, online, peer-reviewed journals covering a wide range of academic disciplines. SCIRP serves the worldwide academic communities and contributes to the progress and application of science with its publication.

Other selected journals from SCIRP are listed as below. Submit your manuscript to us via either submit@scirp.org or Online Submission Portal.
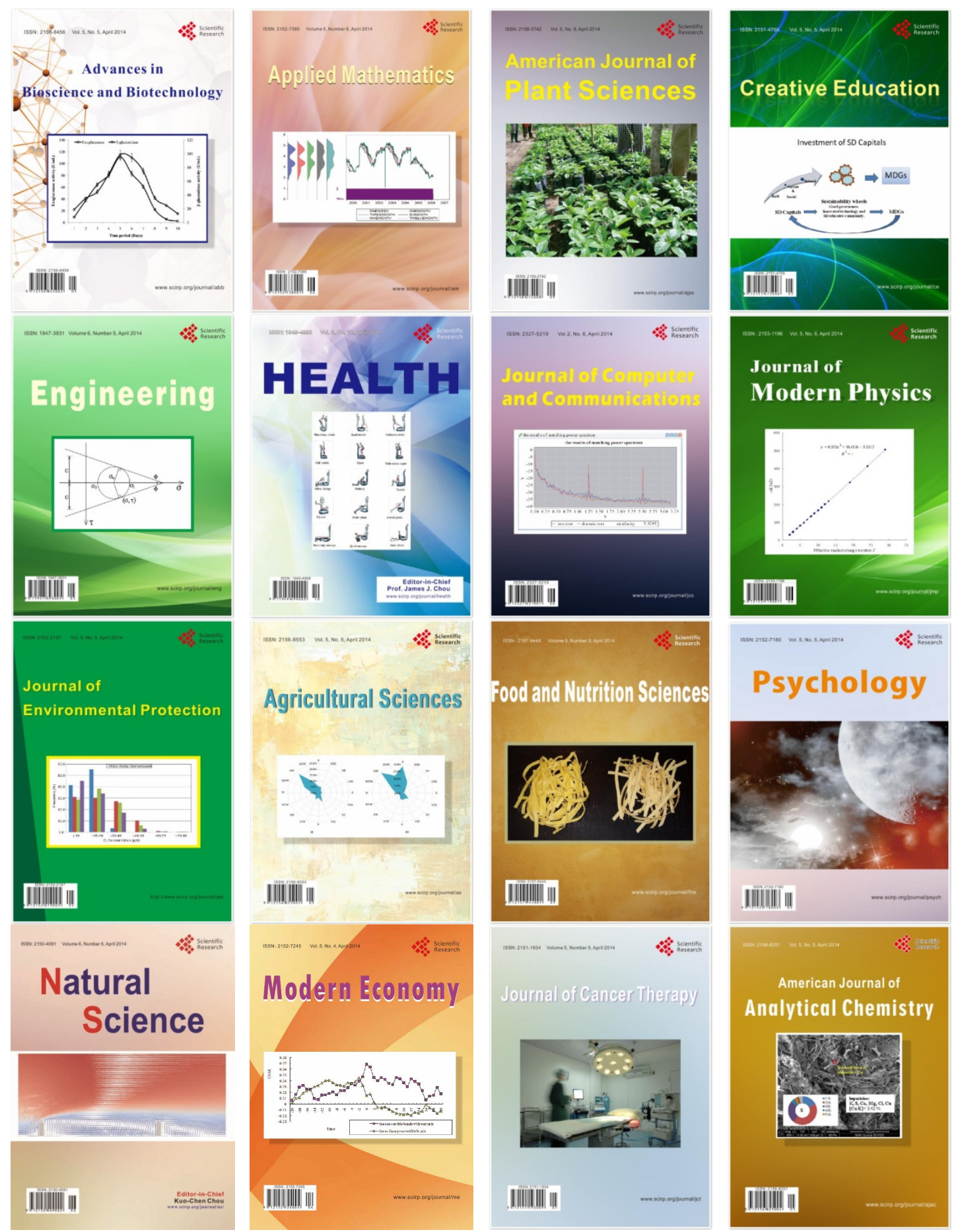